\title{
A way to deal with the fringe-like pattern in VIMOS-IFU data ${ }^{\star}$ (Research Note)
}

\author{
C. Lagerholm ${ }^{1}$, H. Kuntschner ${ }^{1}$, M. Cappellari ${ }^{2}$, D. Krajnović ${ }^{1}$, R. M. McDermid ${ }^{3}$, and M. Rejkuba ${ }^{1}$ \\ 1 European Southern Observatory, Karl-Schwarzschild-Str 2, 85748 Garching bei München, Germany \\ e-mail: clagerho@eso.org \\ 2 Sub-department of Astrophysics, University of Oxford, Denys Wilkinson Building, Keble Road, Oxford OX1 3RH, UK \\ ${ }^{3}$ Gemini Observatory, Northern Operations Center, 670 N. A'ohoku Place, Hilo, HI 96720, USA
}

Received 31 January 2012 / Accepted 12 March 2012

\begin{abstract}
Context. The use of integral field units (IFUs) is now commonplace at all major observatories offering efficient means of obtaining spectral as well as imaging information at the same time. The IFU instrument designs are complex and spectral images have typically highly condensed formats, therefore present challenges for the IFU data reduction pipelines. In the case of the VLT VIMOS-IFU, a fringe-like pattern affecting the spectra well into the optical and blue wavelength regime as well as artificial intensity variations, require additional reduction steps beyond standard pipeline processing.

Aims. We propose an empirical method for the removal of the fringe-like pattern in the spectral domain and the intensity variations in the imaging domain. We also demonstrate the potential consequences of a failure to correct for these effects for any subsequent data analysis. Here we use the example of deriving stellar velocity, velocity dispersion, and absorption line-strength maps for early-type galaxies.

Methods. For each spectrum that we reduce with the ESO standard VIMOS pipeline, we derive a correction spectrum by using the median of the eight surrounding spectra as a proxy for the unaffected, underlying spectrum. This method relies on the fact that our science targets (nearby early-type galaxies) cover the complete field-of-view of the VIMOS-IFU, have spectral properties that vary gradually with position and that the exact shape of the fringe-like pattern is nearly independent and highly variable between neighboring spatial positions. Quadrant-to-quadrant intensity variations are corrected for in an independent step.

Results. We find that the proposed correction methods for the removal of the fringe-like pattern and the intensity variations in VIMOSIFU data-cubes are suitable to permit a meaningful data analysis for our sample of nearby early-type galaxies. Since the method relies on the scientific target properties it is unsuitable for any general implementation in the pipeline software for VIMOS.
\end{abstract}

Key words. methods: data analysis - galaxies: abundances - galaxies: kinematics and dynamics

\section{Introduction}

Integral field units (IFU), combining spectrographic and imaging capabilities, are used to obtain spatially resolved spectra over a typically contiguous field-of-view (FoV). These instruments, which offer an enormous gain in observing efficiency compared to classical long-slit spectrographs, are now used at all major observatories (e.g. GMOS at Gemini, SAURON at WHT, and OSIRIS at Keck). Their often complex instrument designs and the large amount of information on the detectors present challenges for the data reduction. At the VLT, the Visible Multi-Object Spectrograph (VIMOS) has an IFU containing 6400 microlenses coupled to fibres covering the wavelength range 4000-10150 $\AA$ with a set of six grisms (Le Fèvre et al. 2003). With the medium and high-resolution grisms $(R=$ 580-2500), the spectra of a single pseudo-slit will cover the entire length of the CCD, hence only one pseudo-slit can be used. The IFU head is then partially masked by a shutter, so that only a square of $40 \times 40$ fibres is used yielding either a FoV of $27^{\prime \prime} \times$ $27^{\prime \prime}$ at 0.67 per fibre, or a FoV of $13^{\prime \prime} \times 13^{\prime \prime}$ at 0.33 per fibre. The light is fed to four independent spectrographs each covering a quarter of the FoV and recording spectra on its own CCD. The

* Based on observations taken at the La Silla Paranal observatory withing Program ID: 079.B-0402. four identical channels of VIMOS are referred to as quadrants. The VIMOS data reduction pipeline (Izzo et al. 2004; Zanichelli et al. 2005) removes the instrumental signature from the spectra of each quadrant and combines data into a single data cube covering the full FoV.

It has been known for several years (e.g. Jullo et al. 2008, VLT VIMOS manual ${ }^{1}$ ) that spectra acquired by the VIMOS-IFU in conjunction with the "HR-blue" and "HR-orange" grisms exhibit spectral features that are visually similar to fringes. Fringes normally arise from the interference in the CCD detection layer, between incident light and the light reflected from the interfaces of the CCD layers. Given the typical thickness of this layer, fringes are observed at red wavelengths $(>7000 \AA)$. Yet, in the case of the VIMOS-IFU, features (see Fig. 1) resembling fringes are present over the whole wavelength range, suggesting that they are caused by a different mechanism. For the rest of this research note we refer to these features as "fringe-like patterns".

Jullo et al. (2008) investigated the possible origin of the fringe-like pattern, concluding that it originates from either within the mask at the pseudo-slit level or the imperfect position of the masks themselves. However, since the fringe-like pattern

\footnotetext{
1 http://www . eso.org/sci/facilities/paranal/ instruments/vimos/doc; issue 88.0, Sect. 2.8.1.
} 


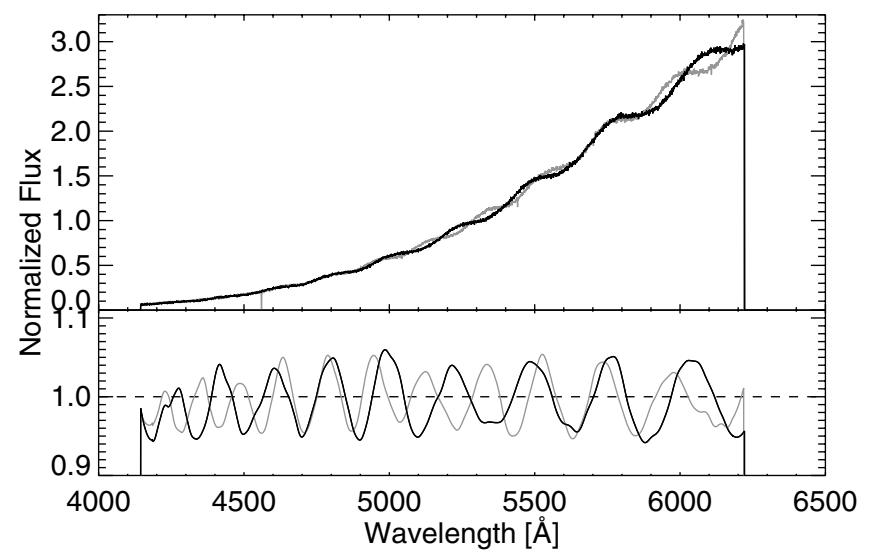

Fig. 1. The fringe-like pattern in the VIMOS-IFU used with the "HRblue" grism. Top panel: flat-field spectrum from a single spatial element (fibre) clearly showing the effects of the fringe-like pattern (black). Bottom panel: the corresponding, normalized correction function for this fibre (black). In gray, we show the flat-field spectrum and correction function from the same spatial element but observed in a different night.

varies between exposures they proposed that the effect probably arises from the flexure of the prisms in the pseudo-slit. The most likely cause of the fringe-like pattern in the spectra is the presence of a "pseudo etalon", of approximately 5-10 micron thickness, associated with the fibre output prism (Dekker, priv. comm.).

In this research note, we present an empirical method for removing the fringe-like pattern, which is implemented as an additional step in the data reduction before stacking individual exposures. We first personally noticed the fringe-like pattern during the analysis of our own VIMOS-IFU observations of nearby early-type galaxies (ETGs) utilizing the "HR-blue" grism (wavelength range 4150-6200 ) with a $27^{\prime \prime} \times 27^{\prime \prime}$ FoV.

For this instrument mode and these specific targets, we developed and tested the method to remove the fringe-like pattern. Our targets are typical of nearby ETGs hence have very smooth surface brightness profiles and cover the entire FoV of the VIMOS-IFU. The full scientific investigation of these ETGs will be presented in a future paper (Lagerholm et al., in prep.). Here we only show our results for the galaxy NGC 3923, which are used to illustrate our correction method.

The paper is organized as follows. Section 2 describes the challenges arising from the data reduction process. In Sect. 3, our method is used to correct the intensity differences and the fringe-like pattern is described. In Sect. 4, we describe the impact of the corrections on the scientific analysis and provide a map of the typical strength of the fringe-like pattern for the VIMOS-IFU. Finally, in Sect. 5 we present our conclusions.

\section{Challenges of the data reduction}

The data reduction was mainly performed with the ESO pipeline (version 2.3.3) using the standard settings described in the pipeline manual ${ }^{2}$. The pipeline processing steps included the subtraction of the median-combined bias image, creation of the spectral extraction mask from a flat-field image taken immediately following the science exposure, and wavelength calibration constructed from the HeArNe lamp exposures. The flux calibration, which is normally performed within the pipeline, did not

\footnotetext{
2 http://www.eso.org/sci/software/pipelines/
}

deliver satisfactory results for some standard stars. We therefore, opted to perform the flux calibration outside the pipeline with IRAF using the standard, sensfunc, and calibrate tasks. This also enables us to combine several standard stars for the flux calibration, which is impossible within the ESO pipeline. The standard stars used for the flux calibration are extracted using the pipeline, where the spectrum is derived by adding several spatial elements thus diminishing the signatures from the fringelike pattern. The final flux calibration curve, which is derived separately for each quadrant, was constructed by fitting a thirdorder polynomial to the standard star data. The low-order polynomial fit ensures that any signatures of the fringe-like pattern are removed.

In the following, we describe three data reduction issues that remain after the standard pipeline procedure: (1) intensity differences between quadrants, (2) intensity stripes across the complete FoV, and (3) the fringe-like pattern in the spectral domain. All three effects, if not accounted for, can significantly affect the scientific analysis, especially when the continuum shape and/or the continuum level play a role.

\subsection{Quadrant-to-quadrant throughput variations}

Owing to the four independent spectrographs used in VIMOS (Le Fèvre et al. 2003), each re-constructed data-cube is composed of four quadrants (Q1 to Q4; see also Fig. 2). After a standard pipeline reduction, the quadrants typically have different intensity levels, as shown in the top left panel of Fig. 2. These differences (of up to $40 \%$ of the peak intensity for our data) give rise to sharp drops/increases in intensity along the connecting quadrant edges. Although these throughput differences between quadrants do not affect the extraction of e.g. kinematics and line-strength measurements for individual spectra, a correction is needed when either a reconstructed image is analyzed or regions across the quadrant edges are binned to reach a specific signal-to-noise ratio $(\mathrm{S} / \mathrm{N})$ requirement.

\subsection{Intensity stripes}

In addition to the quadrant-to-quadrant intensity variations, the reconstructed images from the VIMOS-IFU cubes have diagonal intensity stripes across the FoV (Fig. 2; top left panel). The intensity variations in these diagonal stripes reach up to $\sim 15 \%$ in our data. When comparing individual spectra located at the peak of a given stripe with those next to it, the spectra associated with the stripe have an overall higher flux level over the whole wavelength range. If these stripes were caused by the instrument, one would expect to find discontinuities at the quadrant borders, since the four quadrants originate from independent spectrographs. That the stripes seem to propagate all the way through the quadrant borders implies that they are caused by a pipeline reduction problem, the underlying cause of which is presently unknown.

\subsection{Fringe-like patterns}

More than half of the spectra in a VIMOS data-cube are significantly affected by the fringe-like pattern (see Fig. 1). The amplitude and the frequency of the pattern is not randomly distributed between fibres but shows a clear connection to individual fibre modules. We note that the fringe-like pattern is also present in the raw data, thus is not an artifact created by the extraction process of the spectra. The fringe pattern shifts monotonically inside 

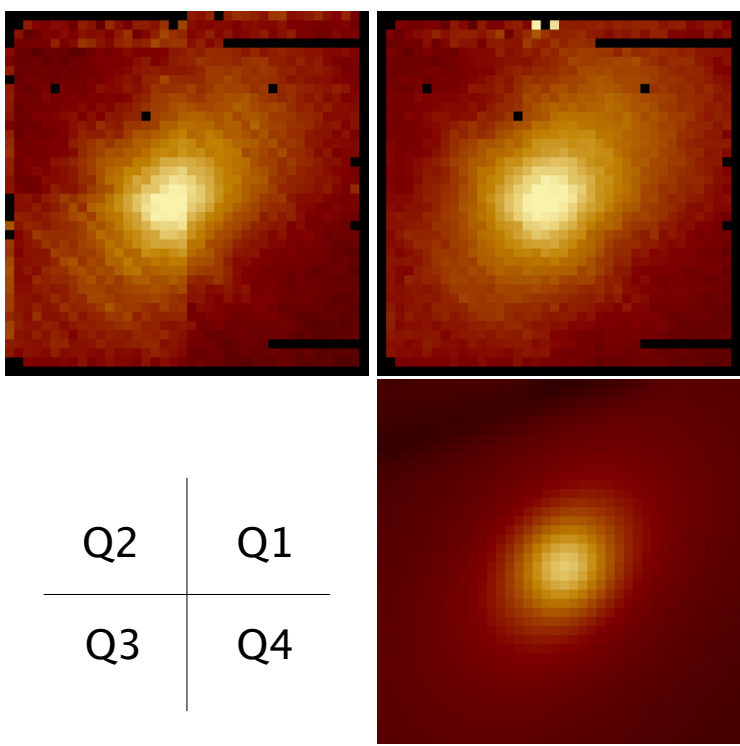

Fig. 2. Top panels: reconstructed images derived from a VIMOS-IFU data cube showing the central $266^{\prime \prime} 8 \times 26$.' 8 of the early-type galaxy NGC 3923. Top left: an example of the image derived from the original data cube as produced by the pipeline. Top right: image derived from the corrected cube. Bottom left: numbering scheme of the four VIMOSIFU quadrants. Bottom right: for comparison, we show an ACS/WFC F606W image of NGC 3923, binned up to the spatial sampling, fieldof-view and broadened to $\sim 1$ '. 2 seeing of the VIMOS data-cube. For the three images of NGC 3923, the orientation is north to the right and east to the top.

a given module but strong breaks appear between fibre modules as illustrated in Fig. 3. In quadrant Q2, almost all spectra are affected, while in the other quadrants fibre modules have a negligible or no fringe-like pattern at all. For each fibre, the fringelike pattern resembles to first order the transmittance function of an etalon with a reflectance of order 1-3\% and a thickness of order 3-10 $\mu \mathrm{m}$ (see Fig. 4 for examples). However, the variation in the amplitude with wavelength and the deviations from a simple etalon transmission function suggest that more than one layer and complex reflectance variations with wavelength may be present in reality.

We note that the fringe pattern varies considerably with time (see Fig. 1; gray lines). Even flat fields taken during the night and adjacent to the science exposure typically do not show the same fringe-like pattern as in the science data. This can be explained by the change in the instrument rotator angle and associated flexure in the instrument. During the science exposure, the rotator angle of the instrument changes in order to follow the target, leading to a different bending of the fibres and therefore a variation in the flexure of the prisms in the pseudo-slit and the fringe-like pattern. The flat-field taken at the end of the science exposure only reflects the pattern at the final rotator angle. Owing to hysteresis effects, returning to the same rotator angle also leads to a different fringe-like pattern (Jullo et al. 2008).

We further note that the observed fringe-like pattern has a frequency and amplitude similar to stellar absorption features in our target galaxy spectra and thus simple filtering with Fourier techniques is unsuitable for removing the effect. For the most affected spectra, the pattern accounts for $\sim 13 \%$ difference in intensity, peak-to-valley (hereafter PTV; see also Sect. 4.3).
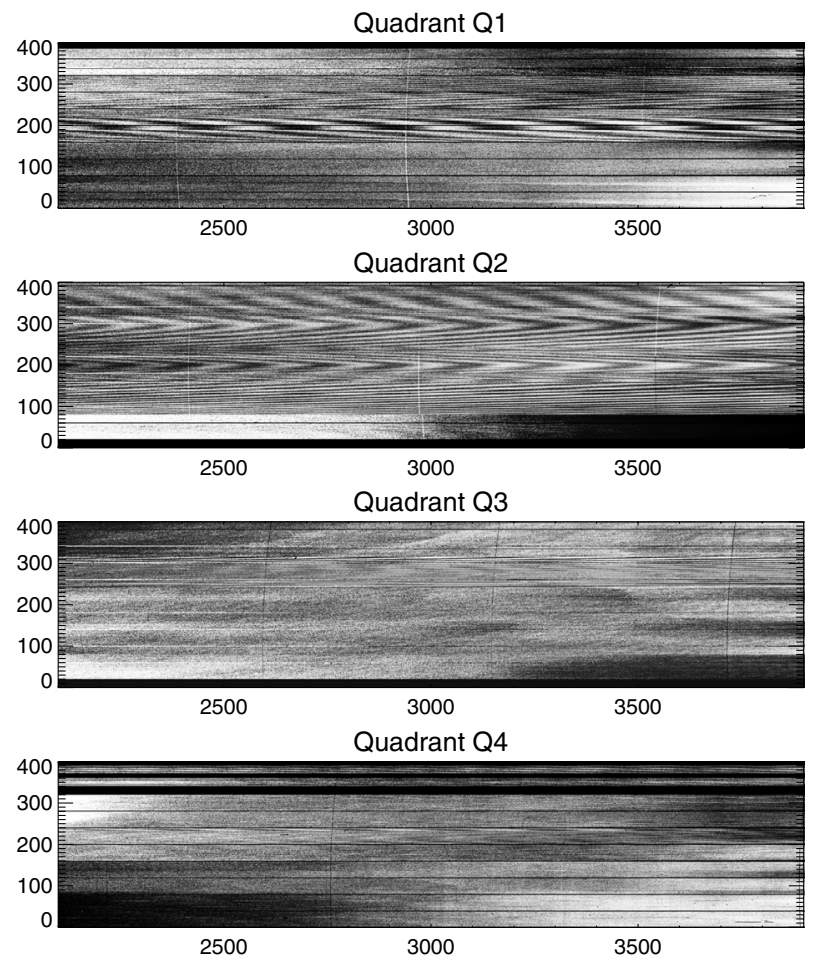

Fig. 3. Variation in the fringe-like pattern for extracted flat-field spectra where the median lamp spectrum has been removed. Each row represents one fibre and wavelength increases from left to right. A subset of the wavelength range, covering pixels 2100 to 3900 and thus corresponding to about $5130 \AA$, to $5675 \AA$, is shown. The example spectrum shown in Fig. 1 is taken from quadrant Q3, where only one fibre module shows a significant fringe-like pattern.

\section{The correction method}

After the extraction and wavelength calibration of spectra using the VIMOS pipeline and flux calibration within IRAF, we correct for the quadrant-to-quadrant intensity differences. We assume that the intensity correction is constant with wavelength and uniform within each quadrant, and re-normalize the quadrants Q1, Q3, and Q4 to quadrant Q2 (see Fig. 2; top right panel). The re-normalization is done by comparing the intensity levels of the neighboring pixels at the quadrant borders and taking a first-order gradient in intensity across the borders into account.

We can now turn our attention to the fringe-like pattern in the spectra and the intensity stripes seen in the reconstructed image. For both, the correction of the fringe-like pattern and the intensity-stripes, the underlying main assumption is that, to first order, the effects are localized, i.e. they are independent among even neighboring spectra. This is a reasonable assumption if the effects are caused by an imperfect fixation of the fibre to the prism (Jullo et al. 2008) creating a "pseudo etalon" associated with the fibre output prism.

For each spectrum in the data-cube, we calculate the median spectrum from the nearest eight spatially surrounding spectra. This median spectrum is, if all eight spectra have different fringe-like patterns, to first order unaffected by the pattern. Since the spectral properties of our targets vary relatively slowly as a function of spatial position and the signal in the neighboring spectra is correlated due to natural seeing effects, this median spectrum can be used as an approximation of the underlying, "true" spectrum in the central pixel. The ratio of the median spectrum to that of the central pixel will provide an estimate of the fringe-like pattern, i.e. a correction spectrum that by 


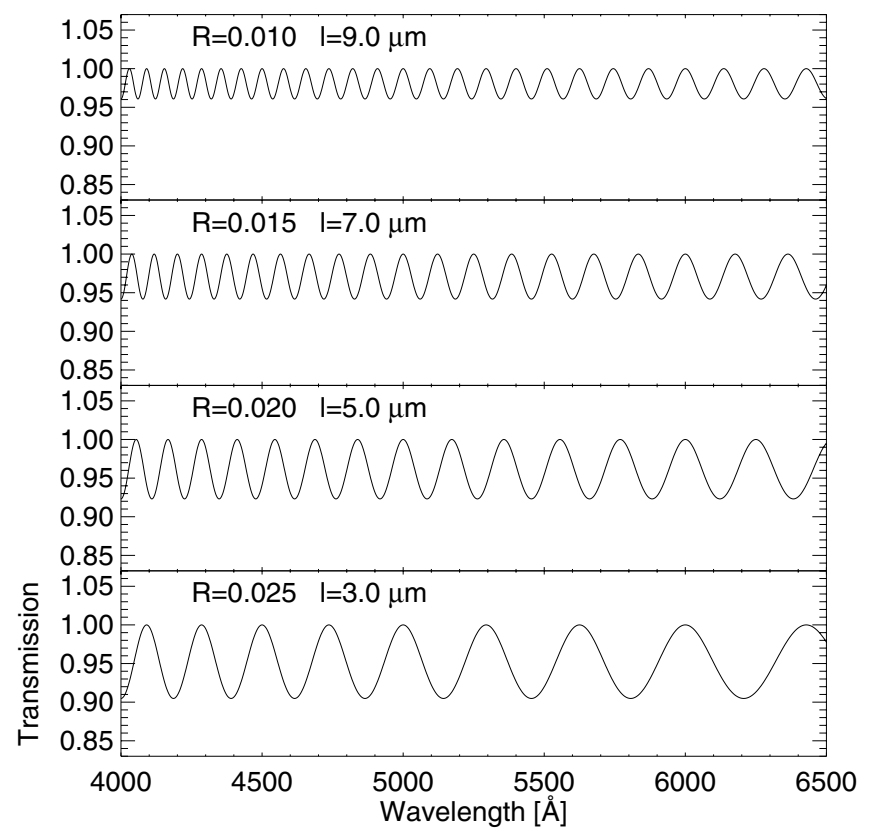

Fig. 4. The transmission of an etalon as a function of wavelength for a few values of reflectance $R$ and etalon thickness $l$. The values are indicated inside each panel.

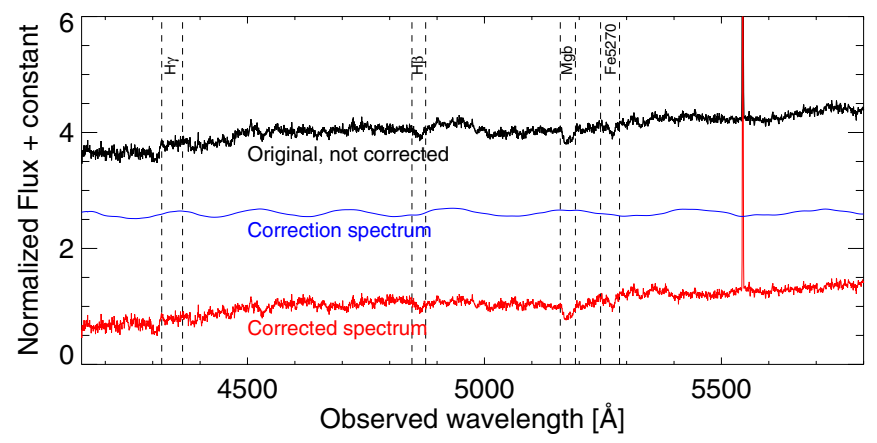

Fig. 5. Example of the correction made for each individual spectrum. Here one of the most affected spectra for the galaxy NGC 3923 (not sky-subtracted) is shown. The top black spectrum represent the original spectrum at one spatial position. The middle blue spectrum shows the correction spectrum derived with our method. The bottom red spectrum shows the corrected spectrum. The black dashed vertical lines indicate the central bandpasses of the line-strength indices $\mathrm{H} \gamma_{\mathrm{A}}, \mathrm{H} \beta, \mathrm{Mg} b$, and Fe5270.

construction has a mean of about one. Owing to the typically limited $\mathrm{S} / \mathrm{N}$ of the correction spectrum we decided to smooth the correction spectrum within IDL using the lowess function, which is part of The IDL Astronomy User's Library (Landsman 1993). We applied a smoothing function using a second-order polynomial for each step of 150 pixels in our case. The resulting correction spectrum preserves the overall shape and amplitude of the fringe-like pattern with negligible noise at smaller wavelength scales (see Fig. 5).

The fringe-like pattern is then removed from the original data-cube by dividing each spectrum by the corresponding smoothed correction spectrum. This procedure is done for all the spectra in the cube, except for the outermost corner spectra and spectra in the neighborhood of regions with dead fibres (shown as black dots or black stripes in the reconstructed IFU image in Fig. 2). Spectra for which we could not derive a correction

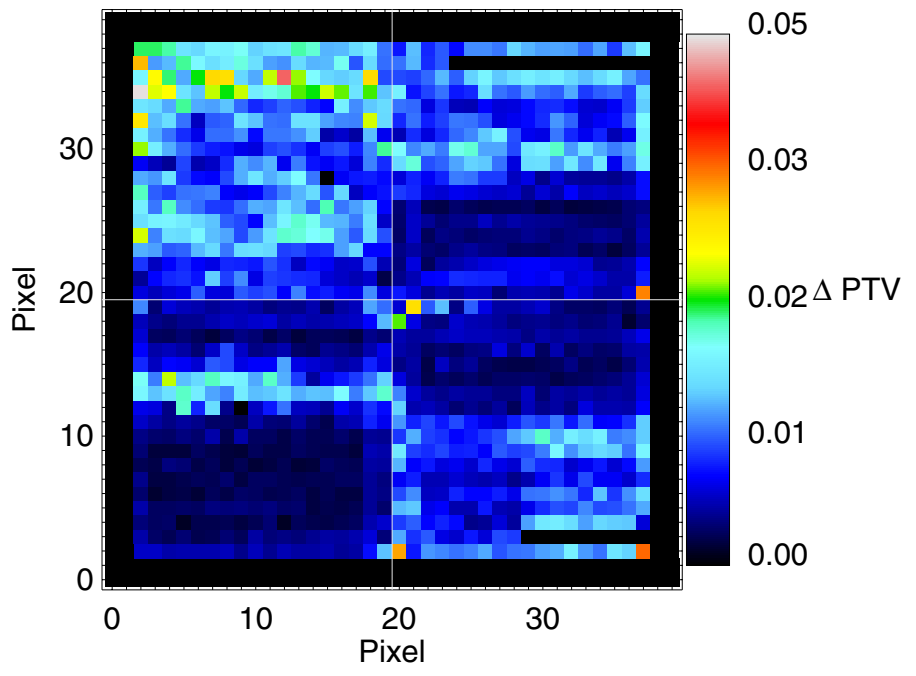

Fig. 6. The maximum differences between the fringe-like patterns, created using both 8 and of the 16 surrounding spectra, in the wavelength range 5188-5620 $\AA$. The white solid lines indicate the borders of the different quadrants. The quadrants are positioned as shown in the bottom left panel of Fig. 2.

spectrum based on the median of at least five or more surrounding spectra were set to zero.

A drawback of the above-described procedure is the effective smoothing that is applied over the scale length of roughly three spatial elements. In particular, at the center of the target galaxies this is a non-desirable effect that has to be accounted for in any subsequent analysis. However, the question arises of whether the median of the eight surrounding spectra provides a good enough estimate of the underlying spectrum or one would need to combine even more spectra to obtain a sufficiently high quality correction spectrum.

To test the effect of increasing the number of spatial elements used to derive the median spectrum, we re-constructed a datacube for an internal flat-field exposure. Here it can be safely assumed that over the scale length of several pixels the underlying, uncontaminated spectrum does not change. In our test, we doubled the number of spectra used for the median spectrum, by considering both the eight surrounding spectra plus eight spectra, equally spaced, from the next closest ring of spectra in the data cube.

Figure 6 shows the maximum difference between the correction spectrum constructed from 8 and 16 neighboring spectra for the whole VIMOS-IFU FoV. Except for a few outliers, especially in the top of quadrant Q2, which show differences up to 5\%, most of the correction spectra show difference of less than $0.6 \%$. We conclude that the median of eight spectra is already sufficient to produce a correction spectrum with an accuracy of about $0.6 \%$. Given that our typical corrections are on the order of $6 \%$ and that we combine several exposures to form the final data cube for analysis, this accuracy appears sufficient. Furthermore, a visual inspection of the differences between taking the median of 8 or 16 spectra indicates that the central wavelength range, containing the most important absorption features, is well-behaved, while extreme differences are often found at the edges of the wavelength range.

\section{Results}

We first describe here the potential consequences of an imperfect data reduction (Sect. 3) for a typical kinematical and stellar 
population analysis of early-type galaxies. We then demonstrate in Sect. 4.2, by using the example of our VIMOS-IFU observations of the ETG NGC 3923, the extent to which the proposed correction method removes the effects. Finally, we provide a map of the VIMOS-IFU FoV showing the regions that are most affected by the fringe-like pattern (see Sect. 4.3).

\subsection{Science impact}

Both the intensity differences and the fringe-like pattern, if uncorrected for, will affect the science derived from the data. When considering the whole spectral range (see Fig. 5), it can be hard to see the effects that the corrections have on the spectra. In Fig. 7, we therefore zoomed into the spectral range of two absorption features, $\mathrm{H} \gamma$ and $\mathrm{Mg} b$, used for the line-strength analysis of the stellar populations in NGC 3923. In the Lick/IDS system (Trager et al. 1998), absorption line-strengths are measured by indices, where a central feature bandpass is flanked to the blue and red by pseudo-continuum bandpasses (see Fig. 7). The mean level of the two pseudo-continuum regions is determined independently on each side of the feature bandpass and a straight line is drawn through the midpoint of each one. The difference in flux between this line and the observed spectrum within the feature bandpass determines the index. In our example, shown in Fig. 7, it is clearly demonstrated that the fringe-like pattern correction changes the continuum level of the spectra on a scale relevant to the determination of the line-strength. In particular, the pseudo-continuum level used in the line-strength analysis, will be located wrongly. For the example spectrum shown in Fig. 5, the $\mathrm{Mg} b$ and $\mathrm{H} \gamma_{\mathrm{A}}$ indices change by $-0.08 \AA$, and $-0.28 \AA$, respectively (uncorrected - corrected spectrum).

The example shown in Fig. 7 represents only one realization of the fringe-like pattern and it can be easily seen how different patterns can lead to a whole range of changes in the line-strength measurements (positive and negative changes). Furthermore, velocity and velocity dispersion measurements can also be affected,although to a smaller extent since the measurements are typically obtained from a wavelength range covering several wiggles/periods of the fringe-like pattern.

To evaluate the effects more quantitatively, we set up simple simulations. The fringe-like pattern is not a simple sinusoidal function, but we approximate it with a sinusoidal with the mean frequency of the fringe-like pattern and vary the phase and amplitude covering the full range seen in the real data. This sinusoidal is multiplied by a fringe-free galaxy spectrum (a galaxy spectrum already corrected for the fringe-like pattern) to simulate the effects of the fringe-like pattern. In our simulations, we changed the amplitude (PTV between $0 \%$ and 15\%) and phase (between 0 and $2 \pi$ ) of the sinusoidal each in seven steps and measured for each spectrum the velocity, velocity dispersion, and line-strengths. The results are summarized in Fig. 8. As expected, the exact location of the fringe-like pattern determines whether a given quantity is changed in a positive or negative direction. Even for large amplitudes, a negligible change is possible when the effects of the fringe-like pattern cancel out. However, the exact phase for this configuration depends on the quantity under consideration.

For a typical amplitude of $5 \%(\mathrm{PTV}=0.10)$, the velocities can be affected by up to $\pm 20 \mathrm{~km} \mathrm{~s}^{-1}$, the velocity dispersion up to $\pm 40 \mathrm{~km} \mathrm{~s}^{-1}$, the $\mathrm{Mg} b$ index by up to $\pm 0.5 \AA$, the $\mathrm{H} \gamma_{A}$ index up to $\pm 1 \AA$, and the Fe5270 index up to $\pm 0.3 \AA$.

Overall, we conclude that for these typical correctionspectrum amplitudes (see also Sect. 4.3), the fringe-like pattern
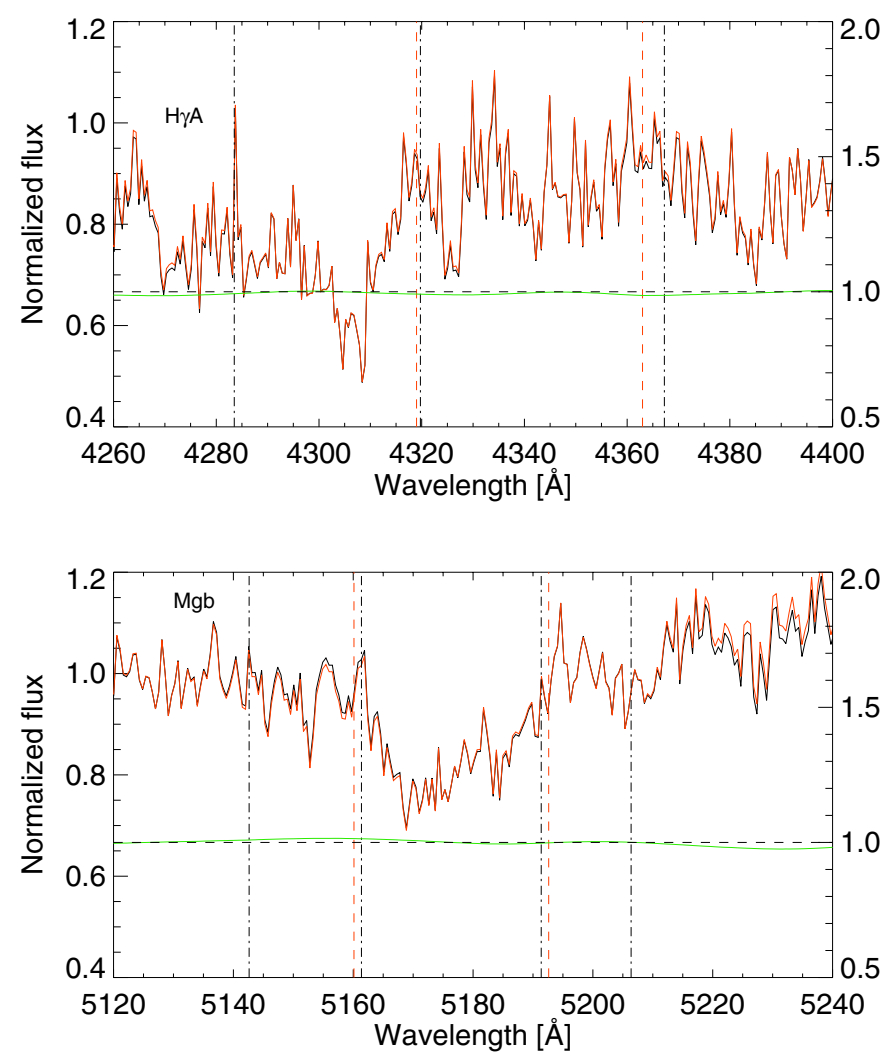

Fig. 7. Difference between a spectrum corrected for the fringe-like pattern (red solid line) and a spectrum that was not corrected (black solid line). The green solid line shows the correction function across the wavelength range where the scaling is given at the right $y$-axis. The line strength is measured between the red dashed lines and the continuum band-passes between the dashed-dotted vertical lines. The linestrength measurements for these two lines in the uncorrected and corrected spectrum are for $\mathrm{H} \gamma_{\mathrm{A}}=-4.79 \pm 0.33 \AA$, and $-5.07 \pm 0.37 \AA$, and for $\operatorname{Mg} b=5.33 \pm 0.28 \AA$, and $5.25 \pm 0.32 \AA$, respectively.

can have a significant influence on the scientific analysis, especially for kinematical and line-strength measurements. We emphasize that the above numbers are only valid for an individual exposure and that the combination of several exposures or fibres will significantly mitigate the problem.

\subsection{Application to our data}

Overall, our correction method provides significant improvements compared to the results obtained from uncorrected data. In the reconstructed image, the intensity differences, both between quadrants and between individual spectra seen as stripes, are now considerably smaller (on average $<5 \%$; see Fig. 2; top left panel). The overall morphology of the science target (e.g. the isophotes) is now in much closer agreement with a direct image, although it does not reach its level of quality (see Fig. 2; bottom right panel).

However, the most important part of the correction is the removal of the fringe-like pattern from the spectra. In Fig. 9, we show the line-strength maps for the $\mathrm{Mg} b, \mathrm{H} \gamma_{\mathrm{A}}$, and Fe5270 indices of NGC 3923, for both the fringe-like pattern corrected and uncorrected cubes. Each cube is the combination of five individual exposures. In the case of the corrected cube, each individual exposure was corrected before combination.

The overall improvement in quality can be directly seen by comparing the uncorrected and corrected line-strength maps in 

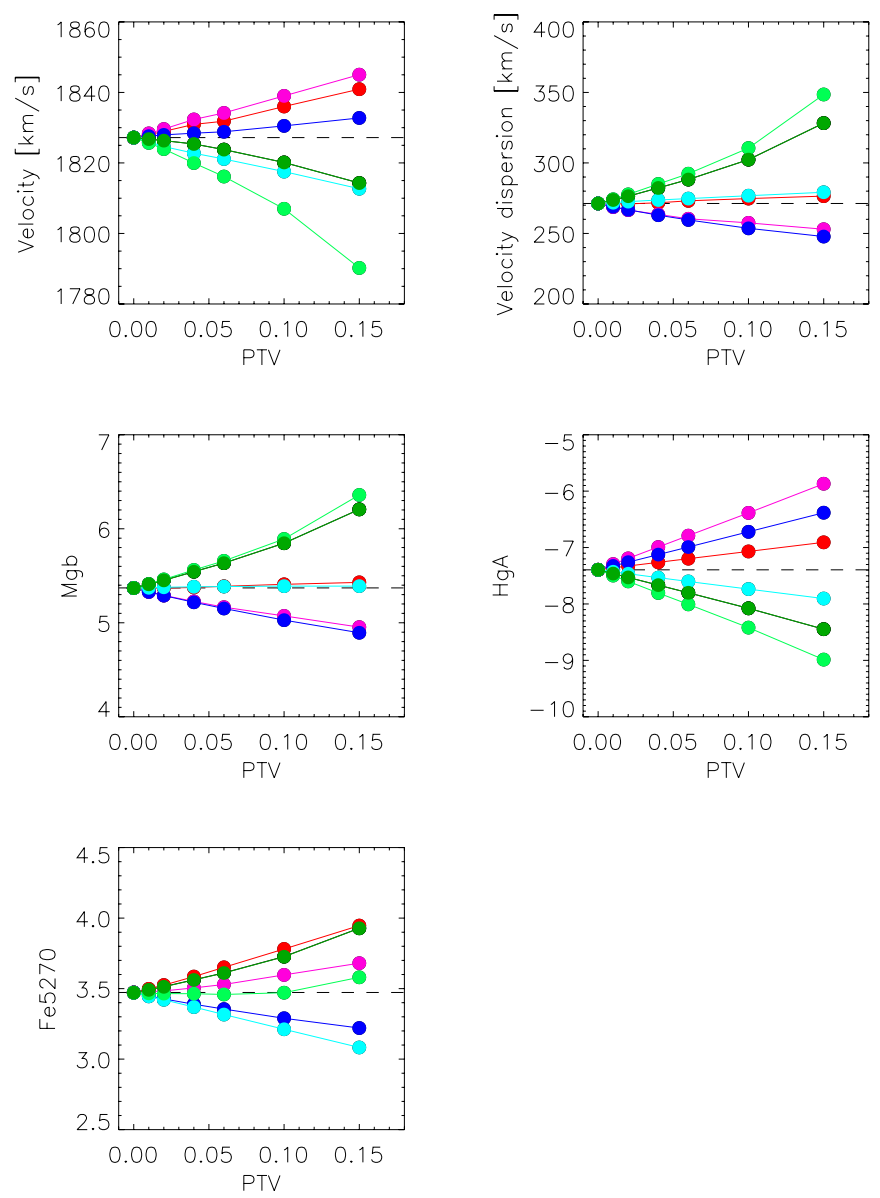

Fig. 8. Changes in velocity, velocity dispersion and the line-strengths, $\mathrm{Mg} b, \mathrm{H} \gamma_{\mathrm{A}}$ and $\mathrm{Fe} 5270$ depending on the PTV of the fringe-like pattern (sinusoidal). Different colors indicates different phase shifts of the sinusoidal, equally separated between 0 and $2 \pi$. The shift in color goes from dark green, red, magenta, blue, light blue, light green, back to dark green. The black dashed lines indicates the correct values given by the fringe free galaxy spectrum.

Fig. 9. The maps constructed with the corrected cubes have metal index values ( $\mathrm{Mg} b$ and Fe5270) that peak toward the center, while the maps for the uncorrected cubes show a more scattered morphology and are not as smooth as expected for an ETG (e.g. Kuntschner et al. 2006). However, the expected differences between the corrected and non-corrected line-strength maps are not as large as one would expect from our simulations described in Sect. 4.1. This can be understood by considering that the final cube was compiled from the combination of five exposures, which helped to average out some of the fringe-like pattern.

The effects of the fringe-like pattern scale with the signal in the data since it is a multiplicative effect. Therefore, a low signal-to-noise exposure, e.g. a sky exposure, will not display a prominent fringe-like pattern. The fringe-like pattern will be hidden in the noise, and we are unable to correct them using our proposed method. However, for the sky subtraction of the galaxies we use a median over the whole sky cube, thus the sky spectrum will essentially be free from the fringe-like pattern.

For science targets for which our proposed method is unsuitable (e.g. low signal-to-noise or highly variable spectral properties across the FoV), one can reduce the fringe-like pattern by averaging several, slightly dithered, exposures. The appropriate number of exposures depends on the science goals and the target itself, but as a general guideline we would recommend obtaining
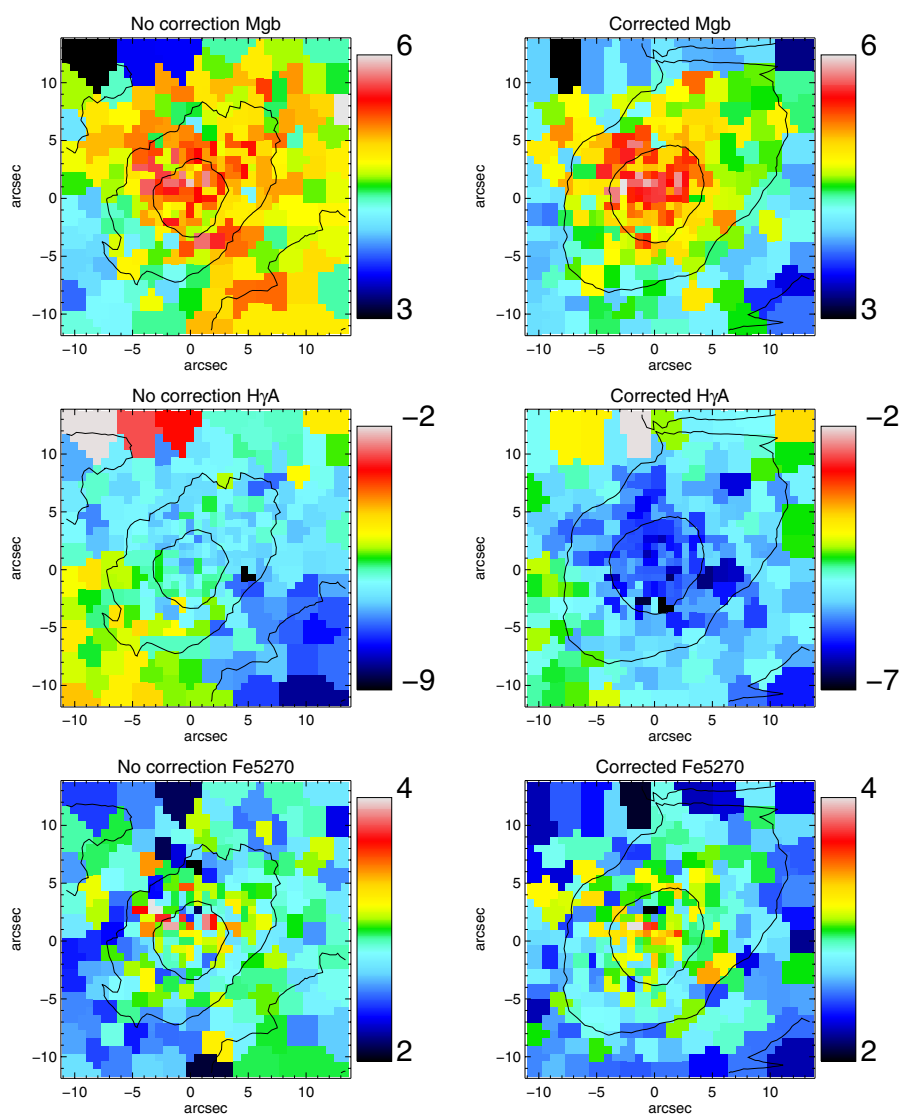

Fig. 9. Line-strength maps for NGC 3923 over $\mathrm{Mg} b, \mathrm{H} \gamma_{A}$, and $\mathrm{Fe} 5270$. In the three plots to the left, the cubes are not corrected and in the three plots to the right the cubes are corrected for the fringe-like patterns. The spectra used for the line-strength analysis are binned, using Voronoi binning (Cappellari \& Copin 2003), in order to have at least a $S / N$ of 60 . The velocities and velocity dispersions are calculated using pPFX (Cappellari \& Emsellem 2004) and are used in the line-strength measurements. The line strengths are measured using the same principle as in Kuntschner et al. (2006).

on the order of eight exposures thereby mimicking the averaging effect of our proposed method.

\subsection{Spatial map of the fringe-like pattern effect}

The four quadrants are not all equally affected by the fringelike pattern - there are variations both between and within the quadrants. Figure 10 shows the maximum PTV values per spatial element, derived from the correction spectra of a flat field. Regions with a prominent fringe-like pattern, connected to individual fibre modules (see also Fig. 3) are clearly visible. While the precise phase and amplitude of the fringe-like pattern varies between exposures, the spatial regions that are significantly affected in a VIMOS-IFU cube can be clearly characterized. For example, quadrant Q2 is the most affected quadrant. The other three quadrants feature regions that are much less or not at all affected. The maximum PTV values in a cube reach 0.13 , while the mean value for the affected fibre models is about 0.06 , i.e. an amplitude of $3 \%$.

\section{Conclusions}

We have shown that additional data reduction steps, to complement the standard ESO data reduction pipeline recipes, can improve the quality of the reduced spectra and therefore 


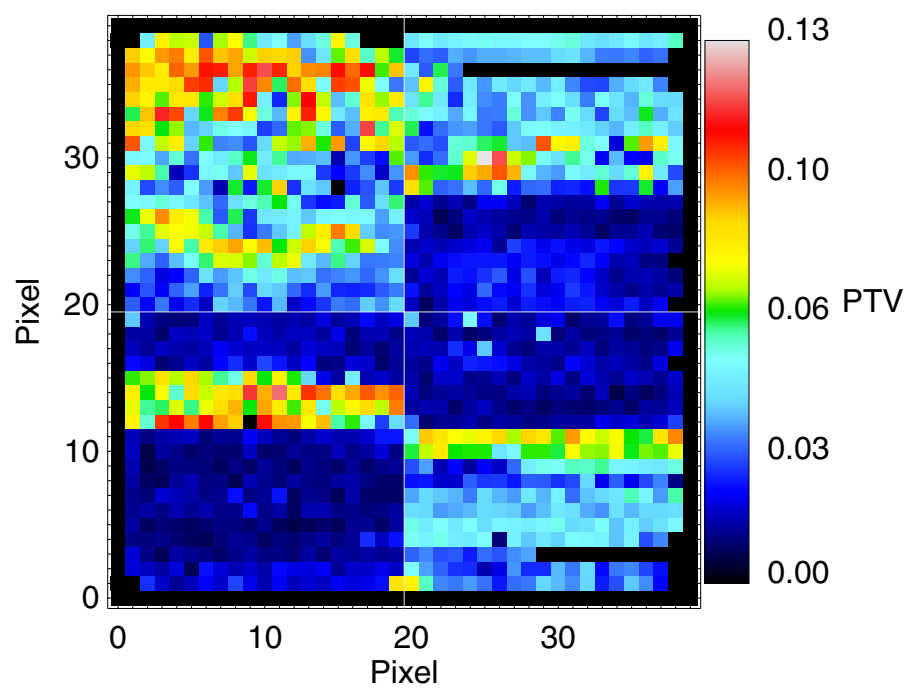

Fig. 10. Spatial map of the maximum variations (PTV) in the correction spectra as derived from a flat-field and the wavelength range 5188-5620 A. Regions with a strong fringe-like pattern are clearly visible and related to certain fibre models (see also Fig. 3). The white solid lines indicate the borders of the quadrants. The quadrants are positioned as shown in Fig. 2, bottom left panel.

the science analysis of VIMOS-IFU data obtained with the "HR-blue" grism. The correction of quadrant-to-quadrant intensity variations and intensity stripes leads to significantly improved reconstructed images. More than half of the VIMOS-IFU spectra obtained with the "HR-blue" grism show, across the full wavelength range, spectral features visually similar to fringes. Unfortunately, the fringe-like pattern is unstable with time and cannot therefore be removed in the flat-fielding process. Our proposed empirical method for minimizing the effects of the fringe-like pattern enables a meaningful kinematical and line-strength analysis for nearby early-type galaxies as demonstrated for the case of NGC 3923. The combination of several exposures into a combined cube also mitigates the fringe-like pattern, although, our newly proposed method working on individual exposures before combination, provides better results. We note, that the proposed correction method is only tested for and will work with objects similar to our science targets (nearby early-type galaxies) that cover the whole VIMOS-IFU FoV and display slowly varying spectral properties over the FoV. When dealing with observations that have strongly varying background or low intensities a different approach should preferably be adopted. In these cases it may be better to rely on the combination of several individual exposures to reduce the effects of the fringe-like pattern or to preform a full modeling of the fringe-like pattern.

Acknowledgements. We would like to thank H. Dekker for useful comments on the possible origin of the fringe-like pattern. We thank the anonymous referee for useful comments. M.C. acknowledges support from a Royal Society University Research Fellowship. R.Mc.D. is supported by the Gemini Observatory, which is operated by the Association of Universities for Research in Astronomy, Inc., on behalf of the international Gemini partnership of Argentina, Australia, Brazil, Canada, Chile, the United Kingdom, and the United States of America.

\section{References}

Cappellari, M., \& Copin, Y. 2003, MNRAS, 342, 345

Cappellari, M., \& Emsellem, E. 2004, PASP, 116, 138

Izzo, C., Kornweibel, N., McKay, D., et al. 2004, The Messenger, 117, 33

Jullo, E., Christensen, L., Smette, A., et al. 2008, in 2007 ESO Instrument Calibration Workshop, ed. A. Kaufer, \& F. Kerber, 343

Kuntschner, H., Emsellem, E., Bacon, R., et al. 2006, MNRAS, 369, 497

Landsman, W. B. 1993, in Astronomical Data Analysis Software and Systems II, ed. R. J. Hanisch, R. J. V. Brissenden, \& J. Barnes, ASP Conf. Ser., 52, 246

Le Fèvre, O., Saisse, M., Mancini, D., et al. 2003, in SPIE Conf. Ser. 4841, ed. M. Iye, \& A. F. M. Moorwood, 1670

Trager, S. C., Worthey, G., Faber, S. M., Burstein, D., \& Gonzalez, J. J. 1998, ApJS, 116, 1

Zanichelli, A., Garilli, B., Scodeggio, M., et al. 2005, PASP, 117, 1271 